\title{
The Effects of Cloud Computing Technology on E-Learning: Empirical Study
}

\author{
Homa Hamidi ${ }^{1}$ and Saeed Rouhani ${ }^{2 *}$ \\ ${ }^{1}$ Department of Information Technology, Mehralborz University, Iran \\ ${ }^{2}$ Department of IT Management, Faculty of Management, University of Tehran, Iran
}

Submission: February 05, 2018; Published: April 13, 2018

*Corresponding author: Saeed Rouhani, Faculty of Management, Jalal ale ahmad highway, Chamran, Tehran, Iran, P0 Box: 1651849661; Tel: +98-21-61117606; Fax: +98-21-77936752; Email: SRouhani@ut.ac.ir

\begin{abstract}
In the world of training, online training is introduced as a modern training services model. For the optimal use of online training services, possession of an appropriate technology platform is vital. Cloud computing is a modern technology which was dramatically interested in the world of information technology, providing software, infrastructure and platform as internet services for the IT world. In this study, the impact of cloud computing on e-learning as an alternative technology for the traditional Web 2, analyzed. The quantitative/experimental method utilized for measurements of two e- learning management servers: one with cloud computing and another with web based technology, with particular QoS (Quality of Service) parameters are done by tools of Apache Benchmark service. Hypothesis testing employed to prove the effects statistically. Three different benchmark tests deployed as follows: analyzing the accessibility to the first page of the Moodle, analyzing the accessibility to the login file system and analyzing the accessibility to the academic file. Final consequences that are resulted from comparing the two servers have shown that the QoS indices of cloud computing server are better than the web 2 based server. The findings prove better performance and quality of service in four aspects of the response time, the throughput passing, the scalability and accessibility. This study grants the E-learning Practitioners and researchers, proved leads, to direct and implement their e-learning systems and platforms on cloud computing infrastructure to achieve identified qualities in their educational and learning services.
\end{abstract}

Keywords : Cloud computing; Quality of service; E-learning

\section{Introduction}

Considering the diverse technologies of education and learning, "Web-based Learning" has more advantages in comparison to "Classroom-based Learning". One of these significant advantages is the reduction of learning costs. Because there is no need to have a physical environment for education and learning, costs are reduced that make learning possible in every time and place that is preferred. In addition, teachers can easily update the educational materials and present the multimedia contents while creating friendly atmosphere that leads to the easier understanding of the learner. At the moment, E-learning systems in their infrastructure level would benefit very little scalability [1]. Nowadays, the world is being faced with rapid changes in educational and enterprise systems that need special solutions. In the age of information Technology, developing with modern technology means to grow and achieve the benefits of the birth of new technology [2]. Technology of "cloud computing" is an option which has been introduced recently for the infrastructure level(s) of e-learning systems in the world of information technology.
Cloud computing is known as the most effective processing sample (mobile processing) [3]. Cloud computing technology has significant impacts on the field of information and communication technology by changing the payment modes and expenses and using software and hardware. Popularity of cloud computing has been widespread due to its two key features [4]:

a. All processing necessities are presented as one service (it can be normally said that everything required is presented in the cloud as a service).

b. The ability to provide computing and processing resources dynamically.

Many researches have been done in the field of cloud computing technology all around the world. And, after investigating numerous articles, cloud computing is introduced as a compatible technology with so many advantages. Analyzing the usage of cloud computing in the e-learning systems is one of theoretical issues that has been addressed in 
several studies. Furthermore, this system has been proposed in the international articles to study, examine and measure the impact of cloud computing on e-learning system.

Therefore, in order to have a better choice and benefit from a desirable technology in the field of e-learning, the following sections of this study analyze and test the influences of cloud computing on the e-learning system profoundly and the main question of this study: "what are the impacts of using cloud computing on the e-learning system?" is going to be answered.

In order to analyze the impacts of cloud computing on the e-learning system in the current study, the impacts of cloud computing has been divided into four criteria: the response time, the throughput, scalability, and accessibility of cloud computing based system in comparison to the web 2 based system. Evaluating the stated criteria can determine what kind of impacts cloud computing has on e-learning system?

At the beginning, the basic definitions and concepts of the present study are going to be introduced in the Theoretical Foundations. The methods applied in the study and the research processes are briefly discussed in the Research Methodology section. In the Data Analysis section, the data of test results are analyzed. In the Discussion section, the main question of this study is answered and the results of the examinations are going to be discussed. And finally, the results of this study are going to be analyzed in the Conclusion.

\section{Theoretical Backgrounds}

\section{The concept of e-learning}

You might have heard a lot of words such as virtual learning, electronic learning, distance learning, web based education, etc. However, the best concept for virtual learning is e-learning. E-learning refers to a set of educational activities using electronic tools such as networks, computers, text, audio, video, etc. [5]. According to Terry Anderson, the learner uses the internet for gaining knowledge, making individual meanings and concepts, increasing learning experiences, achieving learning contents, being connected to other contents, educators and learners and having support and protection while learning [6].

E-learning is a new method of education and its environment has most of the characteristics of a physical environment of an ordinary class. Learners are connected to the teacher via internet and receive the educational programs and contents. This method is a process of teaching and learning using electronic systems and consists of all the education's methods which use electronic tools including audio, video, computer and network and other technologies that are similar. This training method is a new phenomenon in the present world and is somewhat known or still unknown in some other parts of the world. In fact, e-learning is a kind of distance learning.
Most of the limitation of traditional learning is eliminated using e-learning. Therefore, e-learning can be an introduction to achieve some aspects of human rights that is preparing and developing equal opportunities for all the people around the world. As a result, e-learning can not only eliminate the limitations but also the deprivations as well. The main purpose of e-learning is to "learn at every time and place". Saving time, reducing the costs, changing the traditional learning to mobile learning with more motivation and increasing its efficiency are the fundamental reasons for the growth and success of this new method for the development of teaching and learning [7].

The learning management system: The learning management system is a software designed to facilitate teaching. This software is appropriate to make connection among students, professors and officials of the institutes and universities. Also, this system provides the accessibility of students to the presented e-contents using internet and web browsers. The learning management systems are the infrastructures for e-learning and they manage the development and activity of learners [8] and are going to be an available and ubiquitous technology accepted in most of Higher education institute [9]. The LMS software seems to be an ordinary website and the user (student, professor or official) can enter this software using his/her personal information and can perform various operations like observing the courses, participating the online classes, Interactions etc. in this web site using the available choices in his/her user panel and the accessibilities that are given to him/her from the manager.

Using these systems has some advantages for the institutes. Some of the advantages are: Reducing costs of the education, transportation and workshop facilities or the educational course equipment, and reducing students' time-wasting, teaching many students in a short period of time, decreasing the official problems being caused during registration and unit selection. As a result the reduction of costs would come up [10]. Other advantages of these systems are in a way that more students can use these online trainings and we would have increase in the students' abilities and capabilities for better learning. These web based software is appropriate solutions for the validity and analysis of the students' learning by increasing the uniformity and making intensive learning system. Some graphic features like graphs and figures lead to have a more qualitative visual learning. Also, chat rooms, forums and some other similar situations are designed and prepared for increasing the presence of learners in the discussions [11]. This teaching method prepares all the online complementary teaching contents, with no need to go the library, in a way that has significant facilities to be used by teachers and students. This system is expected to present the appropriate lesson in its appropriate time and great quality to learners. Other capabilities of this system are controlling and managing the skills in a wider dimension, analyzing the teaching 


\section{Robotics \& Automation Engineering Journal}

necessities, planning the users' success, giving educational certificates, holding live online courses and/or classes and assigning references [12]. The Moodle software is one of the most popular software systems in learning management that is used in the current experiments of this study. The Modular Object-Oriented Dynamic Learning Environment is electronic teaching software. This program is a web-based free software that the teachers can use the internet for preparing effective environments for online learning. This software system can be installed on a web server, personal computer and/ or a hosting company [13].

\section{Cloud computing concept}

Table 1: Calculations and comparisons via QoS indicators.

\begin{tabular}{|c|c|c|}
\hline $\begin{array}{c}\text { Indicator } \\
\text { time }\end{array}$ & $\begin{array}{c}\text { Indicator formula } \\
\text { Search time+ } \\
\text { web time+ server } \\
\text { time (devoted } \\
\text { to e-learning } \\
\text { management) }\end{array}$ & $\begin{array}{c}\text { Comparing method } \\
\text { Comparing the spent time in } \\
\text { each technology for having } \\
\text { access to a specific source. }\end{array}$ \\
\hline Accessibility & $\begin{array}{c}\text { Number of } \\
\text { operations at a } \\
\text { time }\end{array}$ & $\begin{array}{c}\text { Focusing on the comparison } \\
\text { of the number of requests } \\
\text { to the portal system in each } \\
\text { second. }\end{array}$ \\
\hline MTTF $+M T T R$ & $\begin{array}{c}\text { System is ready and available } \\
\text { for the users for a specific } \\
\text { time in both systems. }\end{array}$ \\
\hline scalability & $\begin{array}{c}\text { The response } \\
\text { time/load }\end{array}$ & $\begin{array}{c}\text { Comparing the response time } \\
\text { to the specific load increase } \\
\text { of the system. }\end{array}$ \\
\hline
\end{tabular}

Cloud computing in its simple definition means: presenting computer services on the internet. Table 1 shows the way users can have access to the services in the cloud. The only thing that is necessary is that your personal computer, cell phone, TV or even refrigerator must have one software connector like one browser to be used for the online cloud services [14].

Cloud computing is a new phenomenon that some of its resource comprising the process unit, memory and the storage section which are not physically presents for the users. Instead, there is a service provider that has and manages these resources in a way that users can have access to them via the internet [4].

\section{Quality of service}

This study has tried to measure the parameters of quality of service or QoS for the cloud computing and web 2 based systems for comparing the performance of these two systems. QoS (Quality of Service) is a service for improving quality of communications. This model consists of some indicators that can measure the quality of one service. The following introduces the four parameters that are considered in this study: the response time, the throughput passing, scalability and accessibility.
The response time: The time that the system needs to react the human requests is called the response time. This parameter is for testing the access to the first page of the Moodle. It is the time the user has to wait to see the first page of the Moodle on the browser. For testing the accessibility to the login system file, it is the time that the user has to wait to enter the portal. And, finally for having access to the course file, it is the time the user has to wait for the course file to be opened. It should be mentioned that in the less the response time of the system, the better performance is resulted [15].

The throughput: Throughput means the successful message delivery rate in a connecting channel on the net or other communicating channels like telecommunications. These information can be delivered using a physical or logical link or pass via a specific network node. Therefore, all these tests that analyze the accessibility to the first page of the Moodle, analyzing the accessibility to the login file system and finally analyzing the accessibility to the course file are the throughput that is equal to the successful message delivery rate in the connecting channels on the internet. It should be stated that in the more the throughput of the system, the better performance is resulted [15].

The scalability: If efficiency of a system hasn't reduced when the number of users increased, the system is scalable. A system with high scalability won't increase significantly the response time facing greater requests. Similarly for each three experiments, with changing amount of requests, scalability of system can be observed and compared with its performance at the time of responding. It is worth mentioning, the higher scalability leads to the more appropriate performance of the system [16].

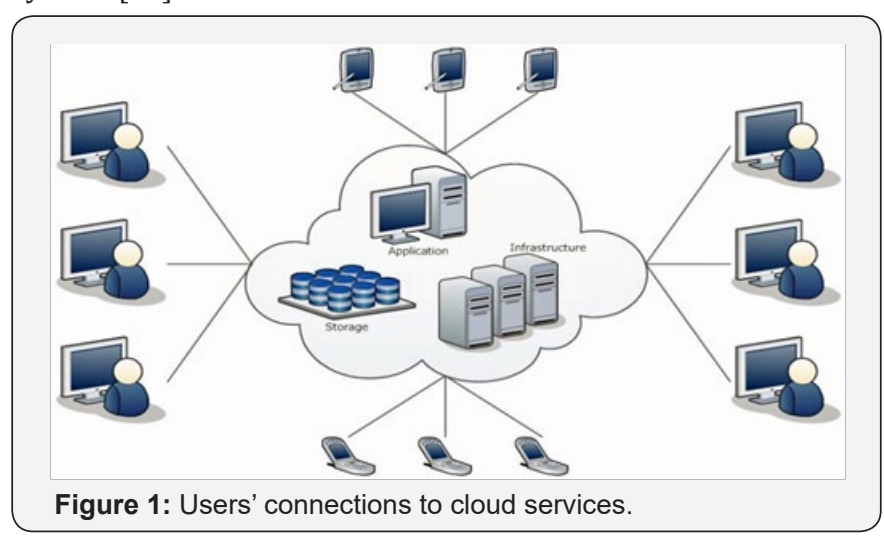

The accessibility: Accessibility is based on the time that the system is ready and available for the users. This parameter is calculated via this formula: A=MTTF/(MTTF+MTTR) MTTF is the working time of the server with no error and MTTR is the time the server can troubleshoot the errors. It should be said that in the more accessibility to the system, the better performance is resulted [17]. 


\section{Robotics \& Automation Engineering Journal}

Figure 1 shows the way the introduced parameters are measured.

\section{Research Methodology}

This study is focused on analyzing the impacts of cloud computing on e-learning in the Electronic Mehralborz University in Tehran. In terms of purpose of this study, analyzing the influence of a new technology in comparison to the traditional present technology and providing the knowledge in the field of applying appropriate infrastructure, is an applicable research. The present study, using management tools and Benchmark Apache Test, compares the performance of LMS system with the web 2 based management system by implementing Moodle on the cloud computing based system. By analyzing the observations and result obtained from the implemented Moodle system on the virtual machine, the QoS indicators like the response time, throughput, accessibility and scalability in comparison to same indicators in the web 2 based system of the Mehralborz University supposed to be tested. The information of Mehralborz system is available via the technical staffs of the university and there are domestic and especially foreign library research and investigations. Therefore, this study has used the quantitative/experimental method.

Mehralborz is the study case for web 2 based system and Amirkabir University system was chosen as the research system for doing the experimental cloud computing system. After analyzing the LMS server of Mehralborz University, a virtual machine with Ubuntu operating system, similar to server infrastructure of Mehralborz University system was chosen. At first, the software Moodle is installed on the cloud computing system. The stated four parameters are studied for comparing the performance of the systems. The Benchmark Apache service was installed on cloud system for measuring the system parameters. Benchmark Apache service is for measuring the HTTP servers. This service helps to show the output service performance by changing the number of requests and loads to the server. In this way, by using ab command and controlling the number of requests and simultaneous requests the output service performance is shown. Three experiments are done to check the exact performance of the Moodle service. The first experiment is to analyze the accessibility to the first page of the Moodle. The second experiment is to analyze availability of the system file's login. And, the third experiment is to analyze availability of the course file. The three parameters of the response time, throughput and scalability are analyzed and tested for each experiment thirty times in different situations of the system load and some data is obtained. Another server is used to research and studies about Mehralborz University with similar infrastructure to the LMS server of Tehran University using Ubuntu operating system that is based on web 2 so they can be compared. Accordingly, the same changes and experiments are done on this server after installing the Benchmark service Moodle.

\section{Research flowchart}

As stated in the previous section, at first, this study analyzes the current technology of web 2 based e-learning system. Therefore, the system is going to be studied precisely. Then, the cloud computing system is going to be simulated. After simulation, the system performance is going to be measured in the new environment. At last, the system efficiency is going to be compared in both environments considering QoS indicators. Figure 2 shows the general framework of the study in a flowchart.

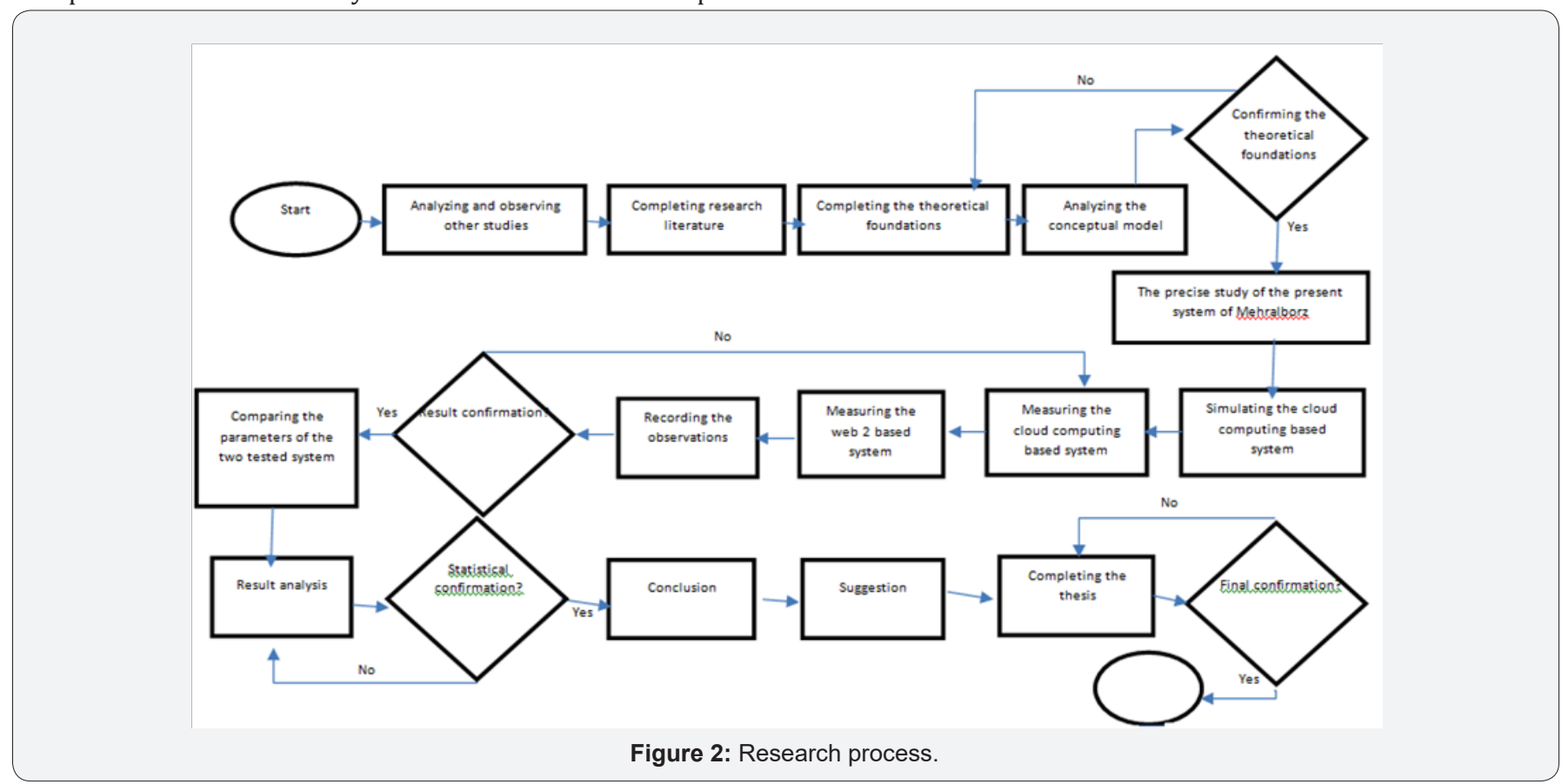




\section{Data Analysis}

After recording the data of the two groups of the web 2 based and the cloud computing, data are analyzed. Then, data normalization is done using the Kolmogorov-Smirnov test, according to the parametric and quantitative characteristics of data, T-test is done. After comparing the response time and throughput parameters obtained from T-test, it is evident that each parameter has two significant differences in each system that shows the higher mean of throughput and lower average response time of the system based on cloud computing in comparison to web 2 based.

The difference between the mean of the throughput system of Mehralborz and Amirkabir in the analysis of having access to the first page of the Moodle is -2518 , in the analysis of having access to the login file system is -1499 , and in the analysis of having access to the course file is -1346 that were measured. Considering the negative sign of the mean differences, the mean of the second group that is the Amirkabir system is higher. Therefore, the throughput server of the cloud computing based system is more than the throughput server of the web 2 based system. The response time parameter is according to what is stated above. The mean difference of the response time of Mehralborz system and Amirkabir in analyzing the access to the first page of the Moodle is 113, the access to the login file system are 66 and 90, and access to the course file is measured to be 92 millisecond. According to the positive sign of the mean difference, the second group is lower that is Amirkabir university system. So, the response time of the cloud computing based server is less that the response time in the web 2 based server. A chart is used for comparing the scalability of these two systems. This chart shows that in all the three experiments, the scalability of cloud computing based system is more that the scalability of web 2 based system. Considering the standard deviation of the response time of these two servers, this scalability is numerically proved as well. The mean of standard deviation in the response time in analyzing the access to the first page of the Moodle for the system of Mehralborz University was 80 and for the Amirkabir system was 14. In analyzing the access to the login file system, these numbers for the cloud computing based system and web 2 based systems were 73, and 23 consecutively. Finally, in analyzing the access to the course file, they were 72 and 12 . The obtained results of these three experiments show more dispersion and changes of response times during the experiment and as a result lower scalability in the Mehralborz system. The performance of the servers and their malfunctions were analyzed to test the accessibility parameter. The malfunctions of the web 2 based system during last year were two times and its downtime was about an hour. The cloud computing based system, benefiting the added servers and balanced load, did not have any malfunction for the last year. Embedding the obtained numbers in the ability to access formula has revealed that the cloud computing based system has had better performance in this parameter and the ability to access has been increased. The ability to access for the web 2 based system was 0.999 and for the cloud computing based system was $100 \%$ during last year.

According to the analysis, the access to the first page of the Moodle, access to the login file system and access to the course file in the Amirkabir system in comparison to the Mehralborz system have been increased in all three experiments. In other words, cloud computing system has had higher throughput, lower response time and higher scalability in comparison to the web 2 based system. The summery of the obtained results are stated in Table 2. According to the results of this study, by doing the three experiments and analyzing the four parameters, we can conclude that the cloud computing technology has been better for e-learning in comparison to the web 2 based system and the mean difference of each of these parameters of throughput, response time and scalability were significant. The ability to access the parameter, as stated above, has been higher in the cloud computing technology due to having more distribution characteristics and more servers.

Table 2: Comparison in performance of two technologies.

\begin{tabular}{|c|c|c|c|c|c|c|c|c|c|c|}
\hline Parameter & \multicolumn{3}{|c|}{ Throughput } & \multicolumn{3}{|c|}{ Response Time } & \multicolumn{3}{|c|}{ Scalability } & Capability \\
\hline Technology & $\begin{array}{l}\text { Page } \\
\text { Moodle }\end{array}$ & Login file & $\begin{array}{l}\text { Course } \\
\text { file }\end{array}$ & $\begin{array}{l}\text { Page } \\
\text { Moodle }\end{array}$ & Login file & Course file & $\begin{array}{l}\text { Page } \\
\text { Moodle }\end{array}$ & Login file & Course file & 1 \\
\hline $\begin{array}{c}\text { Cloud } \\
\text { computing }\end{array}$ & 3063 & 1904 & 1770 & 24 & 36 & 32 & 14 & 23 & 13 & 0000 \\
\hline web 2 & 545 & 404 & 424 & 137 & $\overline{126}$ & $\overline{124}$ & $\overline{80}$ & 73 & 72 & \\
\hline
\end{tabular}

As it can be seen in Table 2, in the experiments to access the first page of the Moodle, the throughput mean of the cloud computing based system in comparison to web 2 based system was $3063 / 545$. For experimenting the login file system this mean was1904/404. And, for having access to the course file experiment, the mean was 1770/424. Therefore, the throughput mean of the cloud computing base system has been significantly higher than the web 2 based system in all the three experiments. The mean of response time in the cloud computing base system in comparison to web 2 based system for the three experiments are measured and consecutively stated as $24 / 137,36 / 126$ and $32 / 124$. Thus, the data show that the response time of the cloud computing based system in comparison to the web 2 based system has been reduced. 


\section{Robotics \& Automation Engineering Journal}

In analyzing the scalability of the systems, the mean of the standard deviation in the response time of the cloud computing based system in comparison to the web 2 based system in all three experiments has been measured to be $14 / 80,23 / 73$, and $13 / 72$. These numbers show fewer changes in the response time of the cloud computing based system when there is increase in the load and as a result we have more scalability in the cloud computing based system in comparison to the web 2 based one. In analyzing the ability to access, as stated above, after analyzing amount of malfunctions in both under-study servers in recent year, the ability to access the cloud computing based system was $100 \%$ and for the web 2 based system was 0.999 that shows more ability to access in the cloud computing based system in comparison to the web 2 base one.

\section{Conclusion}

In the present study, benefiting the infrastructure services of cloud computing, the performance of the learning management system and e-learning system using four parameters of the service quality during three experiments has been measured. According to the measurements, the three experiments have shown higher and better performance of the access to the first page of the Moodle, access to the system file's login and access to the course file. In analyzing the QoS parameters, the cloud computing based system has had higher throughput, lower response time, higher, better scalability and better ability to access in comparison to the web 2 based system. The distributing characteristic of cloud computing and use of balanced load between servers, has had a great influence in the results obtained from the measurements in comparison to the web 2 technology. In fact, the web 2 technology has one central server and the increase of load on the server leads to the significant decrease of the server efficiency. As a result, the cloud computing based system, with its unique performance, has proved the increased efficiency of its systems.

Accordingly, for answering the question "what are the effects of implementing cloud computing on e-learning system?" we can say that the cloud computing technology, being served as the infrastructure of the service, has had better performance in comparison to the web 2 technology for the e-learning systems. Other studies have proved the above statement as well. They have also stated that the cloud computing system has had lower costs. Therefore, the present study, similar to the previous studies, has introduced cloud computing technology as an appropriate and better technology for the e-learning systems.

Despite the importance of security in cloud computing, it has not been possible to measure. So, those parameters are measured that show the performance of each server with the increase in the load. Considering the fact that the cloud computing technology is not completely popular and applicable in Iran, Amirkabir system is chosen. Because it is the only system that shows cloud computing is more meaningful. Also, this system only can present the infrastructure service as a cloud computing service. Thus other cloud computing service including software and platform are not analyzed. In addition to the above statements, due to the lack of integration of the e-learning system, the LMS server of the electronic university has been analyzed and other parts of the e-learning system like TTS are not analyzed in the present research.

Some applicable suggestions are introduced in order to complete the cloud computing based system: the cloud computing technology has some problems with the receiving services that are out of its internal controldue to the Outsourcing the technical controls and infrastructure of the university. Thus, for implementing the cloud computing system more accurately in the electronic universities, it is recommended to have a complete and complex agreement with technical viewpoints after a needs assessment. This agreement must be between the receiving service of the university and the central provider of the cloud computing technology to consider all the necessary aspects of the service. Another subject that can be seen based on the statistical analysis is having the distributed systems in the cloud computing system. This subject is quite effective in the quality of cloud computing based system. Thus, by having more added servers, we can increase the efficiency of the system by providing balanced loads. Off course this option would have more costs for the university without receiving the service form the cloud computing service provider. It is recommended to use private clouds for increasing the security of the information. However, the group clouds that are specified to electronic universities have had complete solutions due to the use of experiences obtained from the e-learning systems around the world. Better usage of cloud computing services needs to have high bandwidth and high speed of the internet. Therefore, those universities that would like to use cloud computing system must have high-speed internet. It is recommended to pay more attention to the geographical distribution of service providers when using cloud computing system which leads to the higher speed to access the server. It should be stated that the satisfaction of the user of service receiver near the geographical zone of the university from its center is important for choosing the service provider center.

\section{References}

1. Vakili G (2014) The usages of the cloud computing technology in the field of Electronic Learning. Iranian Institute of Science and Information Technology.

2. Robinson N, Lorenzo V, Jonathan C, Tony S (2011) The Cloud: Understanding the Security, Privacy and Trust Challenges. RAND Corporation. ed: ISBN.

3. Buyya R, Chee Shin Y, Srikumar V (2008) Market-oriented cloud computing: Vision, hype, and reality for delivering it services as computing utilities. In High Performance Computing and Communications, 2008. HPCC'08. 10th IEEE International Conference on, pp. 5-13. 


\section{Robotics \& Automation Engineering Journal}

4. Gholizadeh (2014) The Intelligent techniques for Risk Management in Cloud Computing. Presented at the The international Conference of Business Development.

5. Morrill D (2011) Cloud Computing in Education. September 12, 2011.

6. Ebadi R (2004) Information Technology and Education.

7. Cappos J, Beschastnikh I, Krishnamurthy A, Anderson T (2009) Seattle: a platform for educational cloud computing. ACM SIGCSE Bulletin, 41 : 111-115.

8. Aljenaa E, Al-Anzi FS, Alshayeji M (2011) Towards an efficient e-learning system based on cloud computing. In Proceedings of the Second Kuwait Conference on e-Services and e-Systems, p. 13.

9. Sclater N (2010) Cloud computing in education. Policy Brief, Unesco Institute for Information Technology in Education.

10. Plummer DC, Thomas J Bittman, Tom A, David WC, Mitchell Smith D (2008) Cloud computing: Defining and describing an emerging phenomenon. Gartner, 17.
11. Dong B, Qinghua Zheng, Mu Qiao, Jian Shu, Jie Yang (2009) Blue Sky cloud framework: An e-learning framework embracing cloud computing. In IEEE International Conference on Cloud Computing, 2009 , pp. 577-582.

12. Alshwaier A, Youssef A, Emam A (2012) A new trend for E-learning in KSA using educational clouds. Advanced Computing 3(1): 81.

13. El-Sofany HF, Al Tayeb A, Alghatani K, El-Seoud SA (2013) The impact of cloud computing technologies in e-learning. iJET 8: 37-43.

14. Liaghat N (2011) Green Technology.

15. Ahmadi M, et al. (2015) Principles of Virtualization and Cloud Computing.

16. Hawkins R (2002) Ten lessons for ICT and education in the developing world. ed, 2002.

17. Bala PS (2010) Intensification of educational cloud computing and crisis of data security in public clouds. International Journal on Computer Science and Engineering 2: 741-745.

\section{Your next submission with Juniper Publishers will reach you the below assets}

- Quality Editorial service

- Swift Peer Review

- Reprints availability

- E-prints Service

- Manuscript Podcast for convenient understanding

- Global attainment for your research

- Manuscript accessibility in different formats ( Pdf, E-pub, Full Text, Audio)

- Unceasing customer service

Track the below URL for one-step submission https://juniperpublishers.com/online-submission.php 